\title{
Unilateral axillary adenopathy with unremarkable breast imaging - differential diagnoses
}

\author{
Gudrun Peters, MD \\ Catherine M Jones, MB BS, BSC, FRCR FRANZCR
}

Regional Imaging Tasmania, Tasmania, Australia

Corresponding author: G Peters (Gudrun.Peters@i-med.com.au)

\begin{abstract}
Unilateral axillary adenopathy may be caused by a wide range of both benign and malignant aetiologies. While the most common cause is inflammation, infection or trauma of the breast, thoracic wall or arm, a significant proportion of cases are due to occult malignancy. In female patients particularly, breast malignancy must be excluded with mammography and ultrasound. Local inflammation, infection or trauma of the thoracic wall or arm should also be sought when deciding the most appropriate recommendation for management. This case report outlines the most significant pathologies and discusses the need to rule out sinister pathology, even when a benign local cause is evident.
\end{abstract}

\section{S Afr J Rad 2012;16(3):104-106. DOI:10.7196/SAJR.702}

A 58-year-old woman was referred for diagnostic workup for lumpiness of her left breast. She had no history of malignancy, systemic disease or recent viral illness. Bilateral mammograms (Figs 1a - d) showed a subtle asymmetrical density in the contralateral (right) breast, that was presumed to be asymmetrical breast tissue. Ultrasound of both breasts demonstrated no suspicious abnormality. The right axillary ultrasound (Fig. 2), however, revealed enlarged nodes with up to $6 \mathrm{~mm}$ cortical thickness but preserved fatty hila.

On examination, the patient's right elbow was markedly inflamed. This had been initially overlooked as the patient's arm was covered during ultrasound. Further history of sub-acute chronic pain and limited range of movement in that joint led to a presumed diagnosis of bursitis. As a local inflammatory cause for the unilateral axillary adenopathy was presumed, no biopsy was performed. Follow-up ultrasound was arranged for 4 weeks later.

\section{Diagnosis}

After discussion with the patient's family physician, she attended for consultation the same day. A clinical diagnosis of elbow bursitis was made and appropriate management commenced, including antibiotics for a presumed superimposed infection. Follow-up ultrasound of the right axilla 4 weeks later revealed that the nodes had returned to normal size and cortical thickness (Fig. 3). The bursitis had resolved in the interval.

\section{Discussion}

Isolated unilateral axillary adenopathy is not an uncommon finding. From the radiologist's perspective, the long list of possible causes can lead to a diagnostic and management dilemma. Axillary adenopathy

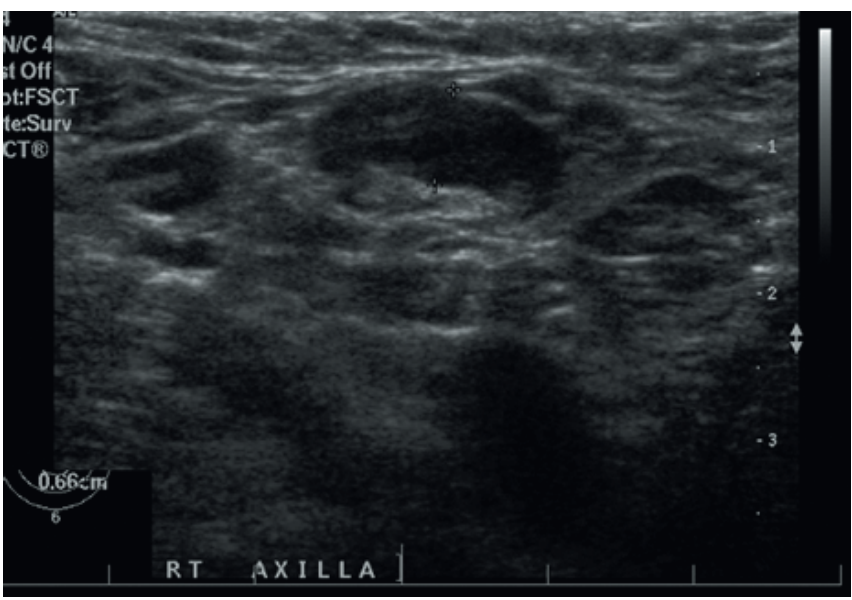

Fig. 2. Ultrasound of the right axilla shows an enlarged lymph node with cortical thickness of $6.6 \mathrm{~mm}$.

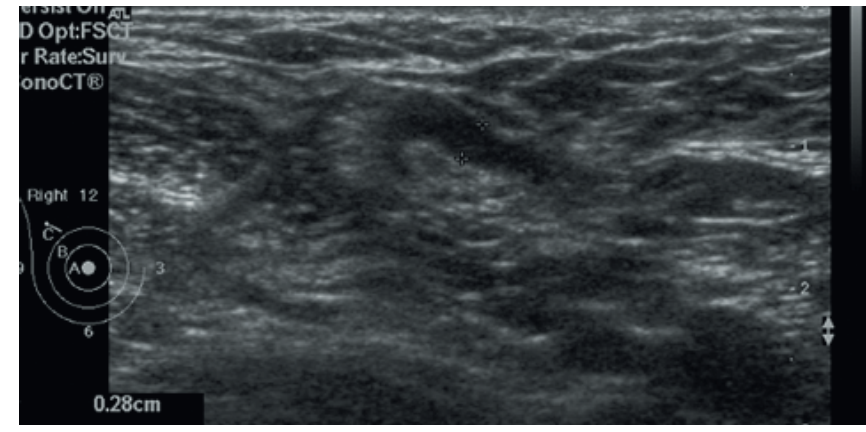

Fig. 3. The follow-up ultrasound 4 weeks later documents that the cortical thickness of the lymph node has returned to the upper limits of normal (2.8 $\mathrm{mm})$.

can be caused by either locoregional or systemic pathology. The breast, thoracic wall and arm have direct lymphatic drainage to the axilla. Local infection, inflammation or malignancy in these regions may lead to unilateral axillary adenopathy. ${ }^{1}$ However, the most probable cause of unilateral axillary adenopathy is non-specific reactivity owing to upper extremity infection or injury. ${ }^{2}$

Systemic illness can less commonly present as unilateral axillary adenopathy. Malignancy such as lymphoma rarely presents as isolated unilateral axillary nodes, ${ }^{2,3}$ although melanoma, sarcoma and other metastatic malignancy may also present in this way. ${ }^{1}$ Auto-immune diseases such as rheumatoid arthritis, lupus, Sjögren's syndrome and dermatomyositis are possible causes. Other causes include sarcoidosis, HIV, Kawasaki's disease, serum sickness and drug reaction. ${ }^{2,4}$ 


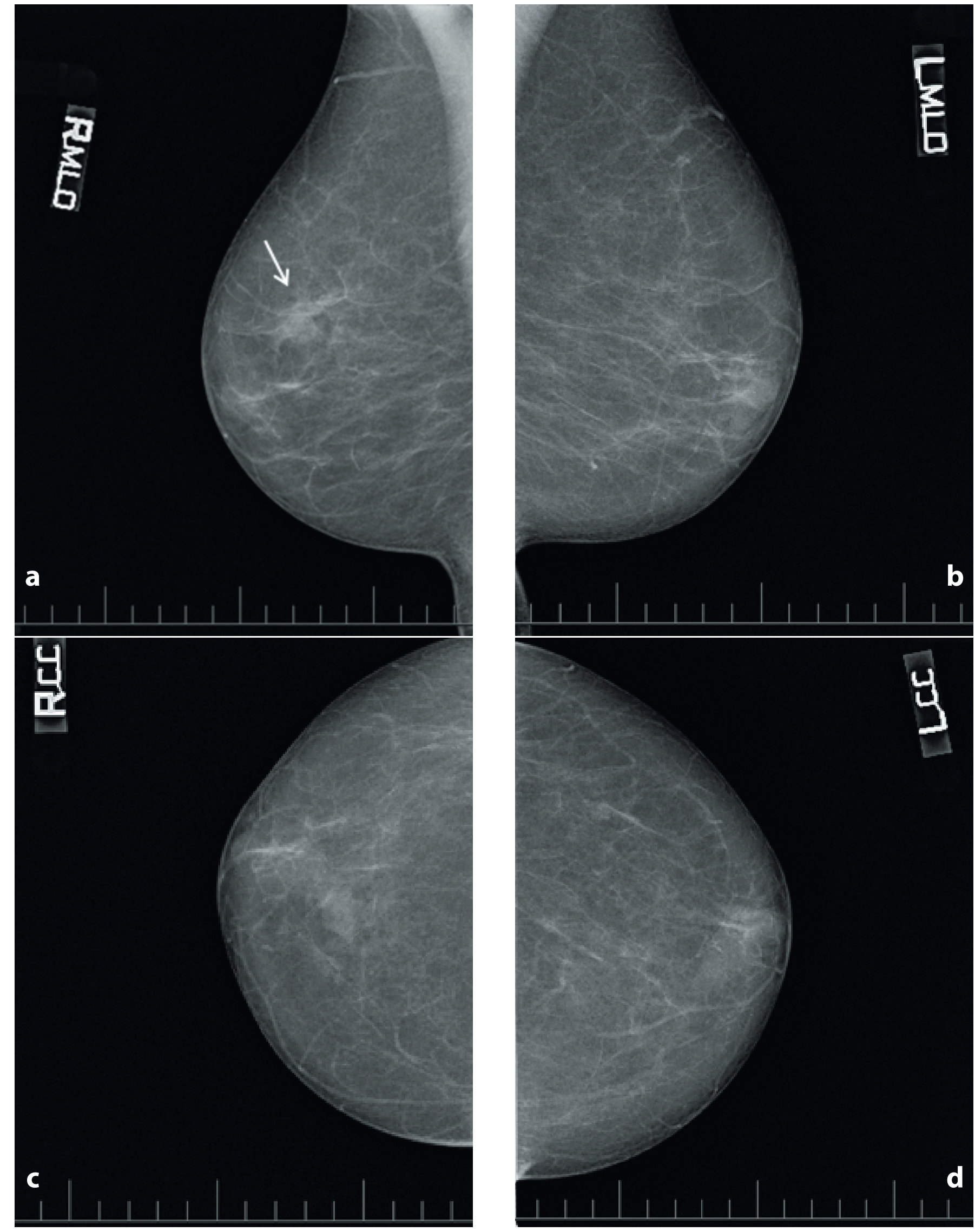

Figs $1 a-d$. Bilateral two-view mammogram shows fatty replaced breast tissue. A small asymmetrical density in the right upper central area of the breast on the mediolateral oblique view (arrow, upper left image) is presumed to be asymmetrical breast tissue. 


\section{CASE REPORT}

In our patient, there was obvious local inflammation which led to the decision to perform short-term follow-up after treatment of the bursitis. As a radiologist, a targeted history and local examination can often elicit a locoregional cause for the adenopathy. Additionally, correlation with previous imaging may give further information about chronicity and stability. If there is evidence of local infection, inflammation or trauma, short-term follow-up may be appropriate.

In patients who have not had recent breast imaging, this should be performed to search for occult breast malignancy, as this is present in up to $12 \%$ of cases. ${ }^{5}$ In patients who have normal breast imaging, nonbreast malignancy is found in $25-33 \%,{ }^{4,6}$ most commonly lymphoma and melanoma. Tissue sampling should be considered if no benign local cause is found. If the decision is made to perform short-term followup, there should be a low threshold for further investigation and tissue sampling if there is no improvement or resolution of the adenopathy.

\section{Conclusion}

Unilateral axillary adenopathy is most frequently due to local inflammation, infection or trauma, and resolves with appropriate treatment. However, in a significant proportion of patients, an underlying occult malignancy is the cause. For this reason, even with local infection or inflammation, short-term follow-up is recommended to ensure resolution. Breast imaging should also be undertaken in female patients to exclude breast malignancy. If the adenopathy does not resolve at follow-up, investigation for a systemic illness such as lymphoma should be performed, and tissue sampling strongly considered.

1. Ferrer R. Lymphadenopathy: differential diagnosis and evaluation. Am Fam Physician 1998;58(6):13131320 .

2. Bazemore AW, Smucker DR. Lymphadenopathy and malignancy. Am Fam Physician 2002;66(11):21032110 .

3. Jose BO, Koerner P, Spanos WJ Jr, et al. Hodgkin's lymphoma in adults - clinical features. J Ky Med Assoc 2005;103(1):15-17.

4. Schwab FD, Burger H, Isenschmid M, Kuhn A, Mueller MD, Günthert AR. Suspicious axillary lymph nodes in patients with unremarkable imaging of the breast.Eur J Obstet Gynecol Reprod Biol 2010;150(1):88-91.

5. Muttarak M, Chaiwun B, Peh WC. Role of mammography in diagnosis of axillary abnormalities in women with normal breast examination. Australas Radiol 2004;48(3):306-310.

6. Brenin DR. The unknown primary tumor presenting with axillary lymphadenopathy. In: Singletary ES, Robb GL, Hortobagyi GN, eds. Advanced Therapy of Breast Disease. London: BC Decker Inc. 2004:659-665. 